\title{
Restoration of sinus rhythm and atrial transport function after the maze procedure: $U$ lesion set versus box lesion set
}

Takashi Nitta, MD, PhD, Yosuke Ishii, MD, PhD, Masahiro Fujii, MD, PhD, Yasuo Miyagi, MD, PhD, Shun-Ichiro Sakamoto, MD, PhD, Atsushi Hiromoto, MD, and Hajime Imura, MD, PhD

\section{ABSTRACT}

Objective: In a U lesion set, the left atrium (LA) roof between the right and left superior pulmonary veins is not ablated, to allow activation to propagate across the posterior LA and to recruit this segment as a contractile atrial component. In contrast, the box lesion set isolates the entire posterior LA.

Methods: To compare the two lesion sets, postoperative freedom from atrial fibrillation (AF) and LA transport function were examined in 402 patients who underwent surgery for AF with a $U$ lesion $(n=329)$ or box lesion $(n=73)$ set. Patients who underwent pulmonary vein isolation alone or other simplified procedures were excluded from the study. LA transport function was quantified at $20 \pm 33$ months postoperatively by the ratio of peak velocity of the A wave to the $\mathrm{E}$ wave (peak $\mathrm{A} / \mathrm{E}$ ) of the transmitral Doppler flow.

Results: In patients with long-standing persistent $\mathrm{AF}$, freedom from $\mathrm{AF}$ was $85 \%$ with the U lesion set and $77 \%$ with the box lesion set at 5 years after the maze procedure, and $82 \%$ and $77 \%$, respectively, at 10 years after the procedure. There was no significant difference between the $\mathrm{U}$ lesion set and box lesion set in patients with long-standing persistent $\operatorname{AF}(P=.30)$ and those with paroxysmal or persistent $\mathrm{AF}(P=.90)$. Proportional hazards analysis identified increased LA diameter $(P=.003)$ and long-standing persistent AF $(P=.03)$, but not the type of lesion set $(P=.51)$, as predictive of postoperative AF recurrence. The postoperative peak $\mathrm{A} / \mathrm{E}$ was significantly greater after the $\mathrm{U}$ lesion set than after the box lesion set $(0.42 \pm 0.22$ vs $0.23 \pm 0.17)$, and multiple regression analysis demonstrated that the type of lesion set and preoperative LA diameter significantly affected postoperative A/E.

Conclusions: The U lesion set restores sinus rhythm frequently as the box lesion set and provides better LA transport function. A dilated LA is a risk factor for postoperative recurrence of $\mathrm{AF}$ and poor postoperative LA transport function.

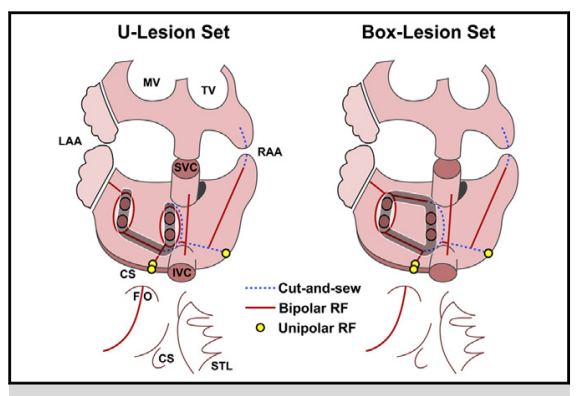

Schemas of the $\mathrm{U}$ lesion set and box lesion set.

\section{Central Message}

The U lesion set restores sinus rhythm frequently as the box lesion set and preserves LA transport function better than the box lesion set.

\section{Perspective}

We compared the U lesion and box lesion sets in terms of postoperative freedom from $\mathrm{AF}$ and LA transport function to determine if the preservation of the posterior LA results in a better postoperative LA transport function. Use of the box lesion set has been shown to decrease the incidence of early atrial arrhythmias. Better-preserved LA transport function was demonstrated with use of the $\mathrm{U}$ lesion set.

See Editorial Commentary page 1070. (J Thorac Cardiovasc Surg 2016;151:1062-9)

Treatment for atrial fibrillation (AF), including any pharmacologic and nonpharmacologic therapies, is performed with the aims of relieving symptoms associated with irregular heartbeat, improving hemodynamics, and

From the Department of Cardiovascular Surgery, Nippon Medical School, Tokyo, Japan.

Read at the 95th Annual Meeting of The American Association for Thoracic Surgery, Seattle, Washington, April 25-29, 2015.

Received for publication May 24, 2015; revisions received Oct 21, 2015; accepted for publication Oct 29, 2015; available ahead of print Dec 2, 2015.

Address for reprints: Takashi Nitta, MD, PhD, Department of Cardiovascular Surgery, Nippon Medical School, 1-1-5 Sendagi, Bunkyo-ku, Tokyo 113-8603, Japan (E-mail: nitta@nms.ac.jp).

0022-5223/\$36.00

Copyright (c) 2016 by The American Association for Thoracic Surgery http://dx.doi.org/10.1016/j.jtcvs.2015.10.108 preventing thromboembolism. The maze procedure has been developed to achieve these goals by restoring sinus rhythm and atrial transport function. ${ }^{1}$ The atrial transport function after the maze procedure is less than optimal, however, owing to the isolation of an atrial component, delayed and desynchronous atrial activation, and possible atrial myocardial ischemia caused by surgical incisions. ${ }^{2}$

Whereas the box lesion set of the maze procedure isolates the entire posterior left atrium (LA) by the LA roof and bottom ablation lines and bilateral pulmonary vein (PV) isolation, in the U lesion set, the LA roof between the right and left superior PVs is not ablated, to allow the activation to across the posterior LA and recruit this segment as a contractile atrial component (Figure 1). Development of bipolar radiofrequency (RF) ablation devices enables 


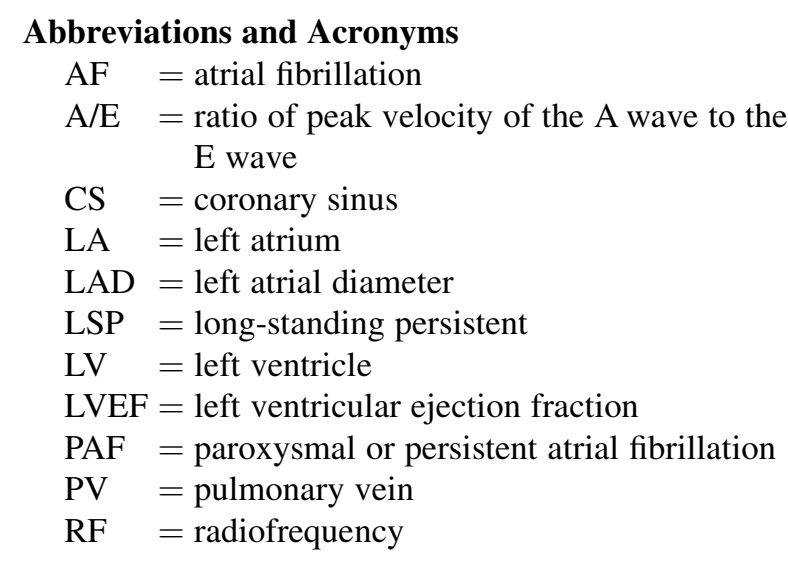

isolation of bilateral PVs without surgical incisions in the posterior LA. ${ }^{3}$

Use of the box lesion set has been shown to decrease the incidence of atrial arrhythmias occurring in the early postoperative period and to reduce the need for antiarrhythmic aganets. ${ }^{4}$ The data suggest that abnormal activations occur in the posterior LA in some patients, as has been documented by intraoperative mapping studies. ${ }^{5}$ Experimental and human studies have demonstrated a better-preserved LA transport function with use of the $\mathrm{U}$ lesion set compared with the box lesion set. ${ }^{6,7}$ The purpose of the present study was to compare the U lesion and box lesion sets in terms of postoperative freedom from $\mathrm{AF}$ and $\mathrm{LA}$ transport function, and to determine whether preservation of the posterior LA results in better postoperative LA transport function.

\section{METHODS \\ Patients}

Between August 1993 and October 2014, a total of 499 patients underwent surgery for AF at our institution. Among these, 402 patients underwent the maze procedure with the $\mathrm{U}$ lesion set $(\mathrm{n}=329)$ or the box lesion set $(\mathrm{n}=73)$, and these patients were included in our analysis. Patients who underwent PV isolation alone or other simplified procedures were excluded from the study. The mean follow-up period was $85 \pm 65$ months (median, 69 months). The patients were evaluated for postoperative cardiac rhythm and restoration of LA transport function, and examined for associations with the preoperative parameters.

Characteristics of the patients who underwent surgery with the U lesion set and those who did so with the box lesion set are compared in Table 1. Preoperatively, left ventricular ejection fraction (LVEF) and left atrial diameter (LAD) were measured in the long-axis view of transthoracic echocardiography. The duration of AF was uncertain in many patients, and was not analyzed in this study. The patients who underwent the maze procedure with the box lesion set had a larger LA and more frequent long-standing persistent (LSP) AF compared with those in whom the $\mathrm{U}$ lesion set was used.

Twenty-one patients had stand-alone AF. More than $90 \%$ of the patients underwent the maze procedure concomitant with other cardiac procedures for structural heart diseases in both groups. Associated structural heart diseases included valvular heart disease in 312 patients (78\%), combined valvular and coronary artery disease in 25 patients, coronary artery disease in 6 patients, and others.

The type of AF was defined based on the Heart Rhythm Society (HRS)/European Heart Rhythm Association (EHRA)/European Cardiac Arrhythmia Society (ECAS) expert consensus statement. ${ }^{8}$ Characteristics of patients with LSP AF and those with paroxysmal or persistent AF (PAF) are compared in Table 2. Compared with the patients with PAF, those with LSP AF had a lower LVEF and a more dilated LA More patients with LSP AF underwent the maze procedure with the box lesion set, whereas the majority of patients with PAF did so with the $\mathrm{U}$ lesion set.

\section{Surgical Procedure}

In all patients, the maze procedure was performed on cardiopulmonary bypass. Patients who underwent an off-pump procedure were not included in the study. The LA appendage was excised and closed in all patients.

Surgical techniques used for PV isolation and at the atrioventricular annuli and coronary sinus (CS) are listed in Table 3. A cut-and-sew technique was used to create a box lesion in 13 patients, a bipolar RF ablation device was used in 59 patients, and cryothermia was used in 1 patient. Cryothermia was used to create a U lesion (bilateral PV isolation) in 84 patients, and a bipolar RF ablation device was used in 245 patients.

A bipolar RF ablation device was used for PV isolation in 304 patients. In this procedure, the LA antrum was clamped with the ablation device and $\mathrm{RF}$ energy was applied; this was usually repeated 3 to 4 times. AF was defibrillated if necessary, and conduction block between the LA and each PV was assessed for by $\mathrm{PV}$ pacing in 203 patients $(66.8 \%)$. The remaining patients did not undergo the PV pacing test because of the possibility of intracardiac thrombi, inability to defibrillate, severely dilated left ventricle (LV), or other reasons. Twelve patients $(5.9 \%)$ were found to have a residual conduction across the PV isolation by the PV pacing test; in these patients, PV isolation was repeated until the complete conduction block was confirmed. After the ascending aorta was clamped and cardioplegic arrest was obtained, at least 1 application of PV isolation was repeated in most patients.

The mitral isthmus and CS were ablated by unipolar RF ablation or cryothermia combined with surgical dissection of the atrioventricular groove. The remaining lesions were created by a cut-and-sew technique, cryothermia, or a bipolar RF ablation device. The cardiac procedures performed concomitantly with the maze procedure are listed in Table 3.

\section{Follow-up and Data Acquisition}

In this retrospective study, the protocol for postoperative electrocardiography (ECG) monitoring did not necessarily follow the follow-up and monitoring guidelines for routine clinical care recommended in the HRS/ EHRA/ECAS expert consensus statement, ${ }^{8}$ because one-half of our patients had undergone surgery before the initial statement was published in 2007. Patients who developed atrial tachyarrhythmias or $\mathrm{AF}$ postoperatively were placed on amiodarone for 3 months. Patients who did not resume sinus rhythm during this period were placed on a beta-blocker with an anticoagulant.

Patients were followed by our outpatient clinic at 6-month intervals. Cardiac rhythm was examined by ECG at every clinic visit and by Holter monitoring for patients with arrhythmias. Recurrence of AF was defined as the first episode of AF or any atrial tachyarrhythmia during the follow-up period. Transthoracic echocardiography was performed in 231 patients, at a mean of $20 \pm 33$ months postoperatively. LA transport function was quantified by the ratio of peak flow velocities of the atrial filling wave (A) to the early filling wave (V) (peak A/E) on transmitral Doppler. The flow velocities were measured from the apical 4-chamber view with the sample volume positioned at the level of the tip of the mitral valve leaflets. ${ }^{6}$ Data from patients with a mitral mechanical valve were excluded from the analysis. 
U-Lesion Set

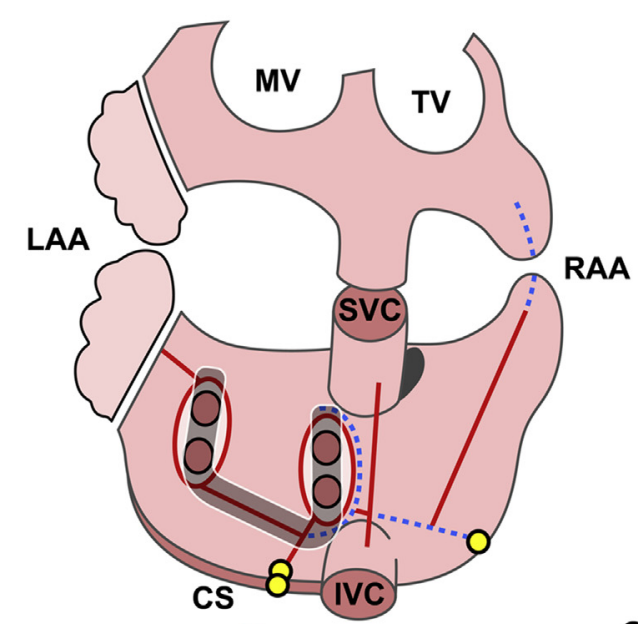

Box-Lesion Set
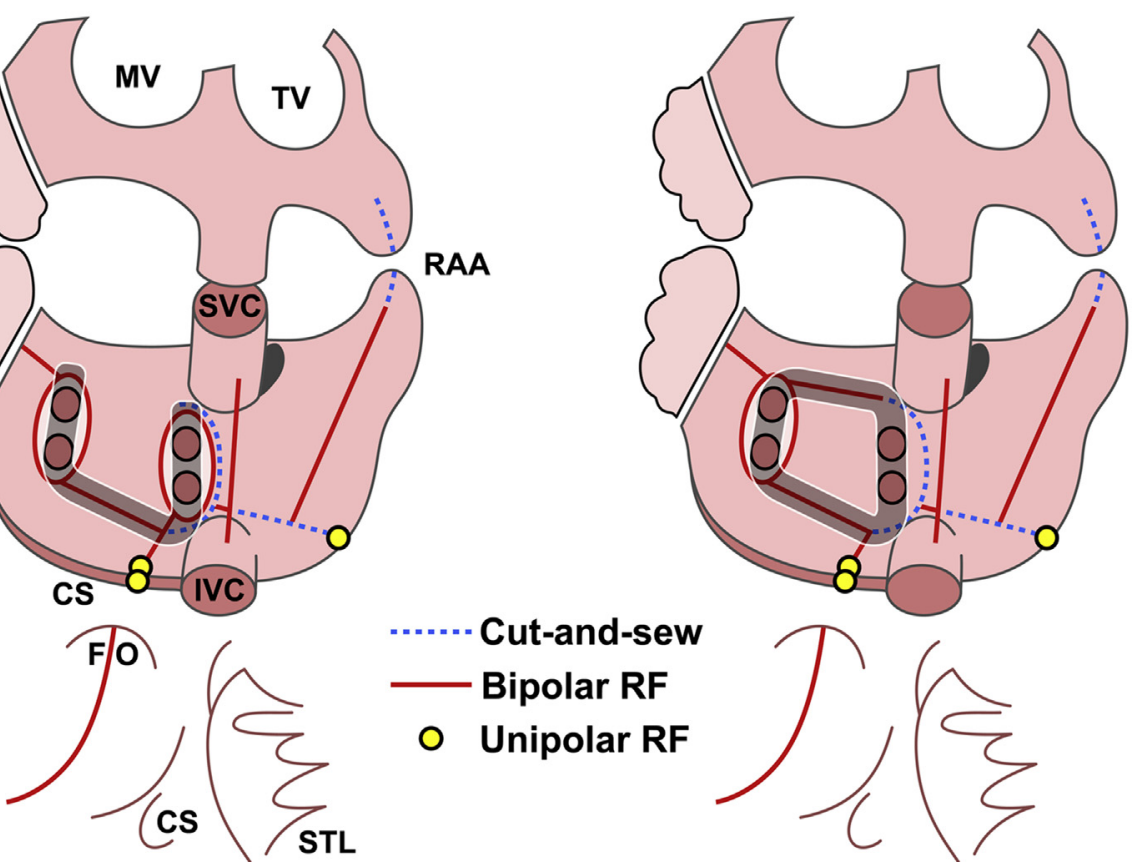

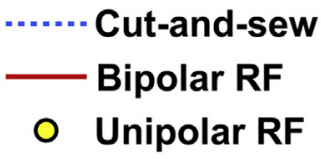

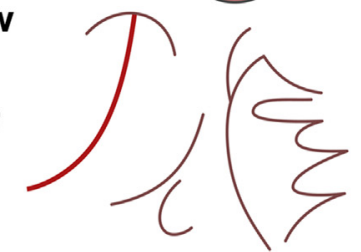

FIGURE 1. The schemas represent the anterior and posterior views of the atria, along with the right atrial septum at the bottom. While the bilateral $P V$ isolation and ablation lines at the $L A$ roof and bottom forms the Box-lesion set and isolates the entire posterior $L A$, the $L A$ roof between the right and left superior pulmonary veins is not ablated to allow the activation to propagate into the posterior $L A$ and to recruit this segment as a contractile atrial component in the U-lesion set. $M V$, Mitral valve; $T V$, tricuspid valve; $L A A$, left atrial appendage; $R A A$, right atrial appendage; $C S$, coronary sinus; $S V C$, superior vena cava; $I V C$, inferior vena cava; $S T L$, septal tricuspid leaflet; $F O$, fossa ovalis; $R F$, radiofrequency.

\section{Data Analysis}

Continuous values are expressed as mean $\pm \mathrm{SD}$. Analysis of variance and the $\chi^{2}$ test were used to compare data between groups as appropriate. Postoperative survival and freedom from AF was examined by the Kaplan-Meier method, and differences between the U lesion set group and box lesion set group were compared using the log-rank test. A Cox proportional hazards model was used to predict parameters for postoperative survival and recurrence of AF. Stepwise multiple regression analysis was performed to analyze the correlation between postoperative peak $\mathrm{A} / \mathrm{E}$ and type of lesion set and the preoperative parameters, including age, sex, structural heart disease, type of AF, preoperative LAD, and LVEF, as independent variables. Bidirectional elimination was performed to

TABLE 1. Patient characteristics

\begin{tabular}{lccc}
\hline \multicolumn{1}{c}{ Characteristic } & $\begin{array}{c}\text { U lesion set } \\
(\mathbf{n}=\mathbf{3 2 9})\end{array}$ & $\begin{array}{c}\text { Box lesion set } \\
(\mathbf{n}=\mathbf{7 3})\end{array}$ & $\boldsymbol{P}$ value \\
\hline Age, year, mean \pm SD & $64 \pm 11$ & $65 \pm 12$ & NS \\
Female sex (\%) & 40.4 & 42.5 & NS \\
LVEF, \%, mean \pm SD & $61 \pm 13$ & $61 \pm 11$ & NS \\
LAD, mm, mean \pm SD & $50 \pm 9$ & $56 \pm 10$ & $<.0001$ \\
LSP AF (\%) & 74.8 & 93.2 & $<.0001$ \\
LA thrombus, n (\%) & $6(1.8)$ & $2(2.7)$ & NS \\
Stand-alone AF, n (\%) & $19(5.8)$ & $2(2.7)$ & NS \\
AF with structural heart & $310(94.2)$ & $71(97.3)$ & NS \\
$\quad$ disease, n $(\%)$ & & &
\end{tabular}

$S D$, Standard deviation; $N S$, not significant; $L V E F$, left ventricular ejection fraction; $L A D$, left atrial diameter; $L S P$, long-standing persistent; $A F$, atrial fibrillation; $L A$, left atrium. identify predictive variables. All statistical analyses were performed using JMP version 12 (SAS Institute, Cary, NC). A $P$ value $<.05$ was considered to indicate statistical significance.

\section{RESULTS}

\section{Mortality and Stroke}

Thirty-day mortality after the maze procedure occurred in 3 patients $(4.1 \%)$ in the box lesion set group and in 7 patients $(2.1 \%)$ in the $\mathrm{U}$ lesion set group, owing to low cardiac output, respiratory failure, LV rupture, acute renal failure, or ischemia of the lower extremities. There was no significant difference in 30-day mortality between the

TABLE 2. Characteristics of patients with LSP AF and patients with PAF

\begin{tabular}{lccc}
\hline \multicolumn{1}{c}{ Characteristic } & $\begin{array}{c}\text { LSP AF } \\
(\mathbf{n = 3 1 4})\end{array}$ & $\begin{array}{c}\text { PAF } \\
(\mathbf{n = 8 8})\end{array}$ & $\begin{array}{c}\boldsymbol{P} \\
\text { value }\end{array}$ \\
\hline Age, year, mean \pm SD & $64 \pm 11$ & $64 \pm 12$ & NS \\
Female sex $(\%)$ & 40.1 & 43.2 & NS \\
LVEF, \%, mean \pm SD & $60 \pm 12$ & $66 \pm 11$ & $<.0005$ \\
LAD, mm, mean \pm SD & $53 \pm 10$ & $45 \pm 8$ & $<.0001$ \\
Concomitant AF surgery $(\%)$ & 94.6 & 95.5 & NS \\
U lesion set $(\%)$ & 78.3 & 94.3 & $<.0001$ \\
\hline
\end{tabular}

$L S P$, Long-standing persistent; $A F$, atrial fibrillation; $P A F$, paroxysmal or persistent AF; $S D$, standard deviation; $N S$, not significant; $L V E F$, left ventricular ejection fraction; $L A D$, left atrial diameter. 
TABLE 3. Intraoperative data

\begin{tabular}{|c|c|c|c|}
\hline Variable & $\begin{array}{l}\text { U lesion set } \\
(\mathbf{n}=\mathbf{3 2 9})\end{array}$ & $\begin{array}{c}\text { Box } \\
\text { lesion set } \\
(n=73)\end{array}$ & $\begin{array}{c}P \\
\text { value }\end{array}$ \\
\hline $\begin{array}{l}\text { Ablation device used for PV isolation, } \\
\mathrm{n}(\%)\end{array}$ & & & $<.0001$ \\
\hline Cut-and-sew & $0(0)$ & $13(18)$ & \\
\hline Cryothermia & $84(26)$ & $1(1)$ & \\
\hline Radiofrequency & $245(74)$ & $59(81)$ & \\
\hline $\begin{array}{l}\text { Device used for mitral isthmus and } \\
\text { CS ablation, } \mathrm{n}(\%)\end{array}$ & & & $<.001$ \\
\hline Cryothermia & $166(50)$ & $21(29)$ & \\
\hline Radiofrequency & $163(50)$ & $52(71)$ & \\
\hline \multicolumn{4}{|l|}{ Concomitant procedure, $\mathrm{n}(\%)$} \\
\hline None & $19(6)$ & $2(3)$ & \\
\hline Aortic valve replacement & $67(20)$ & $12(16)$ & \\
\hline Aortic valve repair & $2(1)$ & $0(0)$ & \\
\hline Mitral valve replacement & $112(34)$ & $29(40)$ & \\
\hline Mitral valve plasty & $136(41)$ & $35(48)$ & \\
\hline Tricuspid valve replacement & $3(1)$ & $0(0)$ & \\
\hline Tricuspid annuloplasty & $113(34)$ & $44(60)$ & \\
\hline Coronary artery bypass grafting & $30(9)$ & $5(7)$ & \\
\hline Atrial septal defect closure & $9(3)$ & $4(5)$ & \\
\hline Repair of other congenital anomaly & $5(2)$ & $0(0)$ & \\
\hline Cardiac tumor & $9(3)$ & $1(1)$ & \\
\hline Septal myectomy & $2(1)$ & $0(0)$ & \\
\hline Left ventricle reconstruction & $2(1)$ & $1(1)$ & \\
\hline $\begin{array}{l}\text { Accessory pathway division } \\
\text { (WPW syndrome) }\end{array}$ & $2(1)$ & $0(0)$ & \\
\hline Ventricular tachycardia surgery & $1(0)$ & $0(0)$ & \\
\hline
\end{tabular}

2 groups $(P=.36)$. During the follow-up period, 14 patients died, at 1 to 85 months postoperatively, due to heart failure, pneumonia, cerebral vascular accident, endocarditis, and unknown causes. Kaplan-Meier estimates of postoperative survival showed a significant difference $(P=.003)$ between the 2 groups of patients; however, a proportional hazards analysis for postoperative survival did not demonstrate any significant difference (Table 4).

Two patients experienced a stroke, at 36 and 83 months postoperatively. Both of these patients had undergone the maze procedure with the box lesion set and were been in sinus rhythm and placed on warfarin therapy for a

TABLE 4. Predictors of postoperative mortality (Cox proportional hazards model)

\begin{tabular}{lccc}
\hline & Hazard ratio & $\mathbf{9 5} \%$ CI & $P$ value \\
\hline Age & 1.00 & $0.94-1.09$ & .93 \\
Female sex & 1.19 & $0.21-6.79$ & .84 \\
LVEF & 0.97 & $0.91-1.03$ & .29 \\
LAD & 0.96 & $0.88-0.99$ & .42 \\
LSP AF & 0.70 & $0.03-1.05$ & .75 \\
U lesion set & 0.20 & $0.13-1.03$ & .13 \\
AF at discharge & 1.15 & $0.06-7.62$ & .90 \\
\hline
\end{tabular}

$C I$, Confidence interval; $L V E F$, left ventricular ejection fraction; $L A D$, left atrial dimension; $L S P$, long-standing persistent; $A F$, atrial fibrillation. mechanical valve. One patient recovered with a mild functional disturbance, and the other died 2 months after suffering the stroke. In both patients, echocardiography did not demonstrate any LA thrombi. No patient in the U lesion set group experienced stroke.

\section{Freedom From AF}

Kaplan-Meier curves of postoperative freedom from AF were compared between the box lesion and U lesion groups in patients with LSP AF (Figure 2) and those with PAF (Figure 3). In the patients with LSP AF, freedom from AF after the maze procedure was $85 \%$ in the $\mathrm{U}$ lesion group and $77 \%$ in the box lesion group at 5 years, and $82 \%$ in the $\mathrm{U}$ lesion group and $77 \%$ in the box lesion group at 10 years. In the patients with PAF, freedom from AF at 5 years after the maze procedure was $98 \%$ and in the U lesion group and $100 \%$ in the box lesion group. There was no statistically significant difference in the incidence of postoperative recurrence of $\mathrm{AF}$ between the 2 groups in the patients with $\operatorname{LSP} \operatorname{AF}(P=.30$, log-rank test $)$ or those with PAF $(P=.90$, log-rank test $)$.

The results of proportional hazards analysis for postoperative freedom from AF are shown in Table 5. The risk for postoperative recurrence of $\mathrm{AF}$ was 3.91 times higher in the patients with LSP AF compared with those with PAF $(P=.03)$. Dilated LA is another significant risk factor for the recurrence of AF; a 1-mm increase in LAD was associated with a 1.05-fold increase in relative risk of AF $(P=.003)$. However, patient age and sex, concomitant surgery for structural heart disease, LVEF, and type of lesion set (box lesion or U lesion) were not statistically significant predictors for postoperative recurrence of $\mathrm{AF}(P=.51)$.

\section{LA Transport Function}

Among the 231 patients who underwent postoperative echocardiography, peak $\mathrm{A} / \mathrm{E}$ measured from transmitral Doppler flow was analyzed in 177 patients without a mechanical valve implanted at the mitral position and without recurrence of AF. Eight patients (19\%) in the box lesion group and 9 patients $(8 \%)$ in the $U$ lesion group showed no A wave $(\mathrm{A} / \mathrm{E}=0)$. Univariate analysis revealed no significant difference in peak $\mathrm{A} / \mathrm{E}$ based on sex, association with structural heart disease, and type of AF, and no correlation between measurement of peak $\mathrm{A} / \mathrm{E}$ and age, preoperative LVEF, or postoperative days; however, peak A/E after the maze procedure was significantly higher in the U lesion group compared with the box lesion group (mean, $0.42 \pm 0.22$ vs $0.23 \pm 0.17$ ), and there was a significant correlation with preoperative LAD. Stepwise multiple linear regression analysis demonstrated that type of lesion set $(t=-3.01 ; P=.003)$ and preoperative LAD $(t=-4.62 ; P<.0001)$ significantly affected postoperative $\mathrm{A} / \mathrm{E}$. The peak $\mathrm{A} / \mathrm{E}$ after the maze procedure is plotted in association with preoperative LAD in Figure 4. 


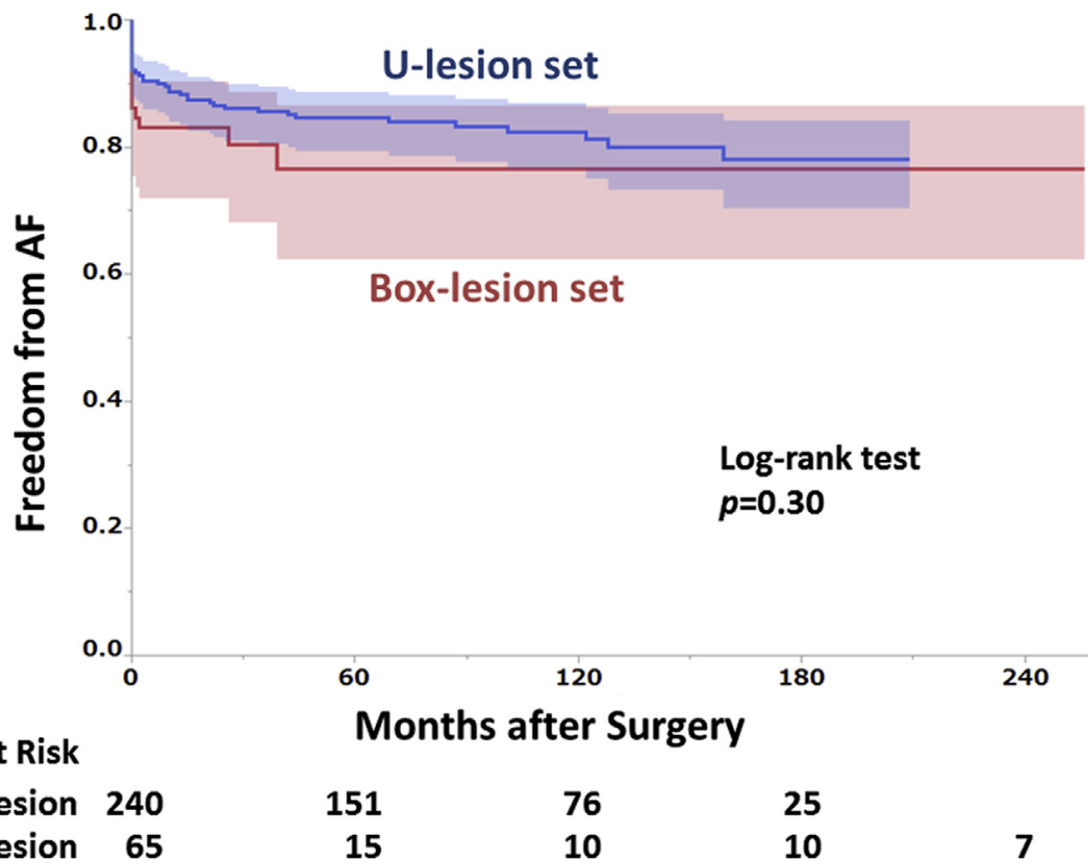

FIGURE 2. Kaplan-Meier curves of postoperative freedom from $A F$ after the maze procedure with U-lesion and Box-lesion set in $L S P A F$ patients. The confidence limits are shown as shaded areas in the graph and the number of patients at risk at the bottom. AF, Atrial fibrillation.

\section{DISCUSSION}

\section{Freedom From AF Recurrence}

The present study did not find a significant difference in the incidence of AF recurrence between the box lesion and $\mathrm{U}$ lesion groups in a long-term postoperative follow-up analysis. Voeller and colleagues ${ }^{4}$ reported a lower incidence of early atrial tachyarrhythmias, greater freedom from AF recurrence, and lower use of antiarrhythmic agents during the early postoperative period in their box lesion group compared with their U lesion group. The discrepant results in their study and the present study may be explained by

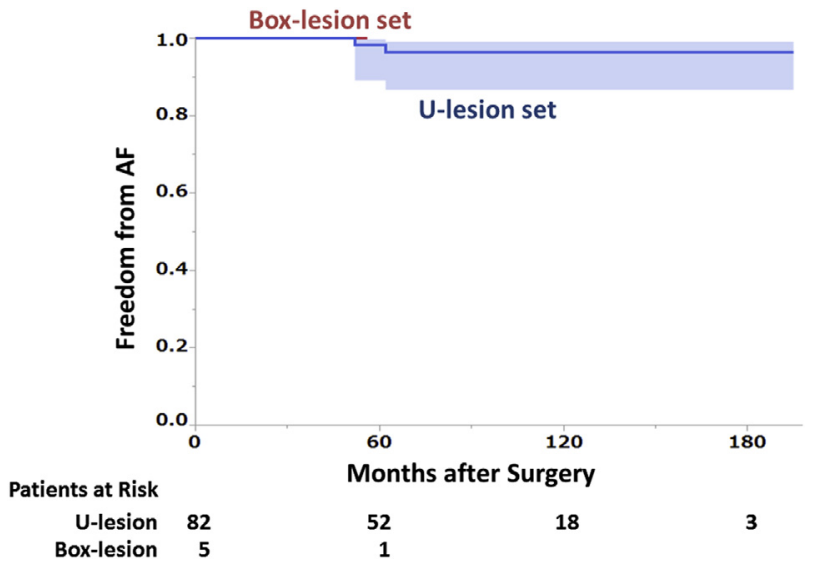

FIGURE 3. Kaplan-Meier curves of postoperative freedom from $A F$ after the maze procedure with U-lesion and Box-lesion set in PAF patients. The confidence limits are shown as shaded areas in the graph and the number of patients at risk at the bottom. $A F$, Atrial fibrillation. differences in patient cohorts and observation periods. In the previous study, more than $60 \%$ of the patients had PAF, compared with $22 \%$ in the present study. In the present study, $95 \%$ of the patients underwent concomitant AF surgery, compared with $64 \%$ in the previous study. Whereas we examined the incidence of $\mathrm{AF}$ recurrence during the mid- to long-term postoperative period, Voeller and colleagues examined the incidence of $\mathrm{AF}$ recurrence during the early postoperative period. In a recent retrospective review study comparing stand-alone $\mathrm{AF}$ surgery and concomitant AF surgery, Lawrance and colleagues $^{9}$ demonstrated that failure to perform PV isolation with a box lesion and early recurrence of atrial tachyarrhythmias were predictive of postoperative $\mathrm{AF}$ recurrence in patients undergoing concomitant AF surgery.

The difference in electrophysiological background between the box lesion set and U lesion set lies in the presence of a conduction block line at the LA roof between the right and left superior PVs. In the box lesion set, the entire posterior LA, including all $4 \mathrm{PVs}$, is electrically isolated by the bilateral PV isolation and the LA roof and bottom lines, thereby confining any focal or reentrant activations arising from the posterior LA to within the region. In contrast, in the U lesion set, the LA roof line is not created, to allow activation to propagate across the posterior LA and to recruit this segment as a contractile atrial component, while allowing a focal or reentrant activation arising from the posterior LA to propagate toward the remaining atria. In an earlier intraoperative mapping study, we reported 2 patients who demonstrated focal activation arising from the 
TABLE 5. Predictors of postoperative AF (Cox proportional hazards model)

\begin{tabular}{lccc}
\hline & Hazard ratio & $\mathbf{9 5} \% \mathbf{C I}$ & $\boldsymbol{P}$ value \\
\hline Age & 1.00 & $0.97-1.03$ & .88 \\
Female sex & 0.63 & $0.30-1.28$ & .20 \\
LVEF & 0.86 & $0.97-1.03$ & .87 \\
LAD & 1.05 & $1.02-1.09$ & .003 \\
LSP AF & 3.91 & $1.13-24.68$ & .03 \\
Structural heart disease & 0.74 & $0.23-4.76$ & .71 \\
U lesion set & 0.75 & $0.34-1.79$ & .51 \\
RF ablation device used & 0.97 & $0.32-3.07$ & .96 \\
$\quad$ for PV isolation & & & \\
RF ablation device used at & 0.85 & $0.36-2.24$ & .73 \\
$\quad$ AV annuli and CS & & & \\
\hline
\end{tabular}

$C I$, Confidence interval; $L V E F$, left ventricular ejection fraction; $L A D$, left atrial dimension; $L S P$, long-standing persistent; $A F$, atrial fibrillation; $R F$, radiofrequency; $P V$, pulmonary vein; $A V$, atrioventricular; $C S$, coronary sinus.

posterior LA out of a total of 50 foci identified in 21 patients. ${ }^{5}$ Those patients may benefit from use of the box lesion set, which isolates the entire posterior LA and blocks propagation of the focal activation arising in the posterior LA.

Complex fractionated electrograms are occasionally recorded at the posterior LA during catheter ablation of persistent AF. ${ }^{10,11}$ These electrograms suggest the presence of substrate for sustenance of $\mathrm{AF}$, and the box lesion set may isolate the substrate in the posterior LA.

The atrial effective mass for sustenance of AF is more reduced with use of the box lesion set than with use of the $\mathrm{U}$ lesion set. The original concept of critical mass hypothesis in AF was described by Garrey, ${ }^{12}$ who observed that the persistence of AF depended on tissue mass and form. The more the atrium is isolated, the less atrial reentry may be

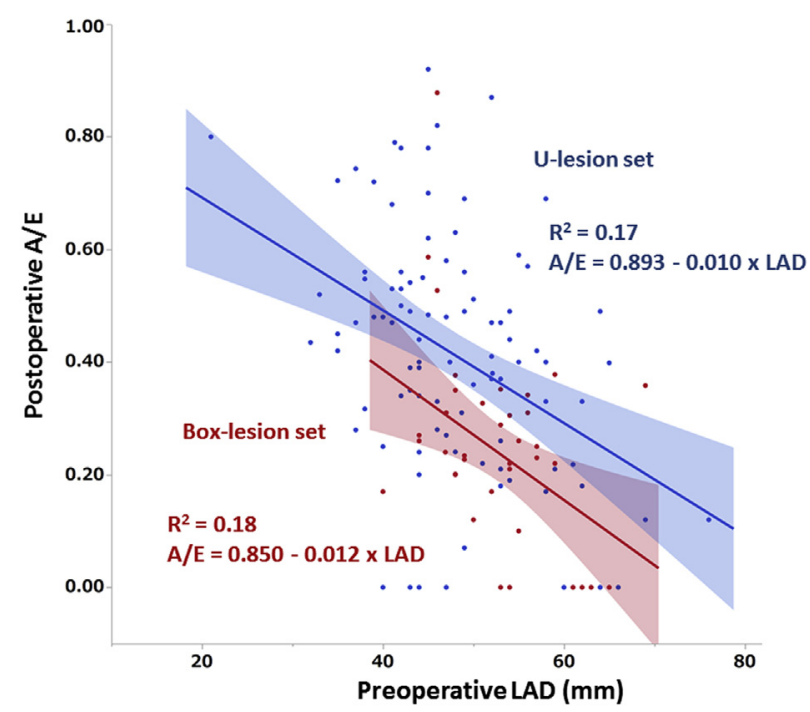

FIGURE 4. The peak $A / E$ after the maze procedure with U-lesion and Box-lesion set was plotted in association with preoperative $L A D$. A/E, Ratio of velocity of the A wave to the $\mathrm{E}$ wave; $L A D$, left atrial diameter. sustained. Lee and colleagues ${ }^{13}$ demonstrated this theory in an experimental study in which the atria were sequentially partitioned using a bipolar RF ablation device.

Although the electrophysiological background described above suggests that the box lesion set may be more effective than the U lesion set in preventing AF, the present study found a similar incidence of postoperative AF recurrence after the maze procedure in the 2 study groups. As shown in Table 1, more patients with LSP AF and a moderately dilated LA underwent the maze procedure with the box lesion set, whereas the majority of patients with PAF and a mildly dilated LA did so with the U lesion set. Our findings suggest that the abnormal activations associated with the posterior LA does not contribute significantly to the sustenance of AF in patients with PAF and a mildly dilated LA, but does so in patients with LSP AF and a severely dilated LA. In a recent randomized trial of catheter ablation for persistent $\mathrm{AF}$ with a mild to moderately dilated LA, Verma and colleagues ${ }^{14}$ found no reduction in the AF recurrence from performing linear ablation or ablation of complex fractionated electrograms in addition to bilateral PV isolation. Arbelo and colleagues ${ }^{15}$ also demonstrated in their prospective randomized study on catheter ablation of PAF that the linear block line at the LA roof was not associated with improved clinical outcomes compared with PV isolation alone.

The lower incidence of AF recurrence in most studies with the box lesion set has been attributed to the LA roof line, which might have functioned as a fail-safe block line against potentially incomplete PV isolation. The abnormal activation originated from the PVs and propagated through an incomplete PV isolation line could be blocked by the LA roof line in the box lesion set. As long as PV isolation is complete, the LA roof line might not be necessary except in the patients whose AF is driven by abnormal activation in the posterior LA. In the present study, every effort was made to achieve complete PV isolation. RF energy was applied at least 3 times in every patient in whom a bipolar ablation device was used for PV isolation. ${ }^{16}$ The conduction block test for PV isolation was performed in the majority of patients, except those in whom the test is contraindicated. In fact, incomplete PV isolation was detected in $5.9 \%$ of patients by the conduction block test despite multiple applications of RF energy, and additional applications of RF ablation were performed in the present study. Furthermore, an additional application of RF ablation was repeated on an arrested heart after the ascending aorta was cross-clamped in most patients. Application of RF energy on a beating heart through a clamp-type bipolar ablation device has proven to be effective and reliable in an experimental study. ${ }^{17}$ The conduction block test is useful to reveal incomplete ablation, ${ }^{18}$ but does not necessarily guarantee conduction block in the long term. Even if PV isolation is verified for complete conduction block intraoperatively, conduction 
between the PV and LA can recover after edema or reversible myocardial damage disappears. ${ }^{19}$ Our meticulous effort to achieve complete $\mathrm{PV}$ isolation might have reduced the rate of $\mathrm{AF}$ recurrence after use of the $\mathrm{U}$ lesion set in the present study.

\section{LA Transport Function}

In the present study, multiple linear regression analysis demonstrated that use of the box lesion set and a dilated LA were associated with decreased postoperative LA transport function. The LA transport function is determined by the amount of effective atrial tissue available for activation, the synchrony of atrial activation, and LV diastolic function. ${ }^{6}$ The amount of effective tissue for atrial transport function is decreased by the LA roof line, which isolates the entire posterior LA. The issue is how much the posterior LA contributes to the transport function and its significance in the prevention of stroke. The posterior LA represents roughly $18 \%$ of the weight of the whole LA in canine hearts ${ }^{6}$ and $29 \%$ of that in human cadaveric hearts. ${ }^{20}$ In addition to the amount of effective atrial tissue, viability of the posterior LA also should affect transport function. Fibrosis has been shown to adversely affect atrial function and increase the risk of stroke in patients with $\mathrm{AF}^{21,22}$

In addition to decreased postoperative LA transport function, a dilated LA also has been identified as a major predictor of postoperative AF recurrence. ${ }^{23,24}$ The present study has also demonstrated that increased LAD and LSP $\mathrm{AF}$ are predictive of postoperative $\mathrm{AF}$ recurrence. Belus and colleagues ${ }^{25}$ have shown that altered active and passive tension generation at the sarcomere level is an aspect of contractile dysfunction and may contribute to the self-perpetuation of AF and development of atrial dilatation in chronic human AF. The present study failed to demonstrate a significant correlation between preoperative LA size and postoperative LA transport function; however, there was a mild linear correlation $(r=0.38)$ between preoperative LA diameter and postoperative peak $\mathrm{A} / \mathrm{E}$ in the patients who underwent the maze procedure with the $\mathrm{U}$ lesion set, suggesting that preservation of the posterior LA may support resumption of greater LA transport function postoperatively.

\section{Clinical Implications}

Although there was no significant difference in the incidence of postoperative AF recurrence between the $\mathrm{U}$ lesion and box lesion groups, our previous mapping study identified a few patients with abnormal electrical activity in the posterior LA that could be isolated using the box lesion set. At the same time, isolation of the entire LA impairs recovery of the LA transport function to some extent. To balance these conflicting effects, intraoperative mapping may be useful to identify any focal activations arising in the posterior LA and determine the optimal lesion set to use for each patient. Rolf and colleagues ${ }^{26}$ took a tailored atrial substrate approach based on low-voltage areas in addition to bilateral circumferential $\mathrm{PV}$ isolation in catheter ablation of AF. They found low-voltage areas at preferred sites in $10 \%$ of their patients with PAF and in $35 \%$ of those with persistent AF. These patients are considered candidates for use of the box lesion set.

\section{Limitations}

Because our protocol for postoperative ECG monitoring did not necessarily follow the guidelines of the HRS/ EHRA/ECAS expert consensus statement, ${ }^{8}$ the accuracy of postoperative assessment might be inconsistent among patients who underwent surgery in different periods. Postoperative follow-up with 24-hour Holter monitoring should be performed consistently at 6-month intervals to assess for recurrence of AF.

Moreover, echocardiograms were recorded in different postoperative periods throughout the study. We previously reported a serial change in atrial transport function after the maze procedure. ${ }^{7}$ Transport function increased gradually during the first 3 postoperative months and was maintained for at least 12 months. In the present study, echocardiography was recorded at a mean of $20 \pm 33$ months postoperatively, after initial functional recovery was completed.

In this study, LA transport function was quantified by the peak $\mathrm{A} / \mathrm{E}$ of the transmitral Doppler flow measured by echocardiography. The early and atrial filling waves of the transmitral Doppler flow are significantly affected by LV diastolic function, LA volume, and other parameters. The majority of the patients had valvular heart disease, with varying degrees of LV diastolic function. Patients with a mechanical valve at the mitral position were excluded from the analysis; however, patients with a bioprosthetic valve were included. Transmitral Doppler flow may differ when measured at the tip of a bioprosthetic valve, a repaired valve, and a native valve. A different modality for measuring a more intrinsic LA transport function is needed to validate the preservation of the posterior LA in the U lesion set.

Finally, this is a retrospective observational study with nonrandomized selection of the lesion set used in the maze procedure. Patients with a severely dilated LA and LSP AF were inclined to undergo the maze procedure with the box lesion set. Further studies, including a prospective randomized controlled study, are needed before definitive conclusions can be drawn.

\section{CONCLUSIONS}

The U lesion set restores sinus rhythm frequently as the box lesion set and provides improved LA transport function. 
A dilated LA is a risk factor for postoperative recurrence of $\mathrm{AF}$ and poor postoperative LA transport function.

\section{Conflict of Interest Statement}

All authors have nothing to disclose with regard to commercial support.

\section{References}

1. Cox JL, Schuessler RB, D'Agostino HJ Jr, Stone CM, Chang BC, Cain ME, et al. The surgical treatment of atrial fibrillation, III: development of a definitive surgical procedure. J Thorac Cardiovasc Surg. 1991;101:569-83.

2. Nitta T, Lee R, Schuessler RB, Boineau JP, Cox JL. Radial approach: a new concept in surgical treatment for atrial fibrillation. I: concept, anatomic, and physiologic bases and development of a procedure. Ann Thorac Surg. 1999;67: 27-35.

3. Prasad SM, Maniar HS, Schuessler RB, Damiano RJ Jr. Chronic transmural atrial ablation by using bipolar radiofrequency energy on the beating heart. $J$ Thorac Cardiovasc Surg. 2002;124:708-13.

4. Voeller RK, Bailey MS, Zierer A, Lall SC, Sakamoto S, Aubuchon K, et al. Isolating the entire posterior left atrium improves surgical outcomes after the Cox maze procedure. J Thorac Cardiovasc Surg. 2008;135:870-7.

5. Nitta T, Ishii Y, Miyagi Y, Ohmori H, Sakamoto S, Tanaka S. Concurrent multiple left atrial focal activations with fibrillatory conduction and right atrial focal or reentrant activation as the mechanism in atrial fibrillation. J Thorac Cardiovasc Surg. 2004; 127:770-8.

6. Nitta T, Lee R, Watanabe H, Harris KM, Erikson JM, Schuessler RB, et al. Radial approach: a new concept in surgical treatment for atrial fibrillation, II: Electrophysiologic effects and atrial contribution to ventricular filling. Ann Thorac Surg. 1999;67:36-50.

7. Ishii Y, Nitta T, Fujii M, Ogasawara H, Iwaki H, Ohkubo N, et al. Serial change in the atrial transport function after the radial incision approach. Ann Thorac Surg. 2001;71:572-6.

8. Calkins H, Kuck KH, Cappato R, Brugada J, Camm AJ, Chen SA, et al. 2012 HRS/EHRA/ECAS expert consensus statement on catheter and surgical ablation of atrial fibrillation: recommendations for patient selection, procedural techniques, patient management and follow-up, definitions, endpoints, and research trial design: a report of the Heart Rhythm Society (HRS) Task Force on Catheter and Surgical Ablation of Atrial Fibrillation. Heart Rhythm. 2012; 9:632-96.

9. Lawrance CP, Henn MC, Miller JR, Sinn LA, Schuessler RB, Damiano RJ Jr. Comparison of the stand-alone Cox-maze IV procedure to the concomitant Cox-maze IV and mitral valve procedure for atrial fibrillation. Ann Cardiothorac Surg. 2014;3:55-61.

10. Atienza F, Calvo D, Almendral J, Zlochiver S, Grzeda KR, MartínezAlzamora N, et al. Mechanisms of fractionated electrograms formation in the posterior left atrium during paroxysmal atrial fibrillation in humans. J Am Coll Cardiol. 2011;57:1081-92.

11. Lee G, Roberts-Thomson K, Madry A, Spence S, Teh A, Heck PM, et al. Relationship among complex signals, short cycle length activity, and dominant frequency in patients with long-lasting persistent AF: a high-density epicardia mapping study in humans. Heart Rhythm. 2011;8:1714-9.

12. Garrey WE. The nature of fibrillatory contraction of the heart: its relation to tissue mass and form. Am J Physiol. 1914;33:397-414.

13. Lee AM, Aziz A, Didesch J, Clark KL, Schuessler RB, Damiano RJ Jr. Importance of atrial surface area and refractory period in sustaining atrial fibrillation: testing the critical mass hypothesis. J Thorac Cardiovasc Surg. 2013;146:593-8.

14. Verma A, Jiang CY, Betts TR, Chen J, Deisenhofer I, Mantovan R, et al Approaches to catheter ablation for persistent atrial fibrillation. $N$ Engl J Med. 2015:372:1812-22.

15. Arbelo E, Guiu E, Ramos P, Bisbal F, Borras R, Andreu D, et al. Benefit of left atrial roof linear ablation in paroxysmal atrial fibrillation: a prospective, randomized study. J Am Heart Assoc. 2014;3:e000877.

16. Wakasa S, Kubota S, Shingu Y, Kato H, Ooka T, Tachibana T, et al. Histological assessment of transmurality after repeated radiofrequency ablation of the left atrial wall. Gen Thorac Cardiovasc Surg. 2014;62:428-33.

17. Gaynor SL, Ishii Y, Diodato MD, Prasad SM, Barnett KM, Damiano NR, et al Successful performance of Cox-maze procedure on beating heart using bipolar radiofrequency ablation: a feasibility study in animals. Ann Thorac Surg. 2004; 78:1671-7.

18. Gersak B, Kiser AC, Bartus K, Sadowski J, Harringer W, Knaut M, et al Importance of evaluating conduction block in radiofrequency ablation for atrial fibrillation. Eur J Cardiothorac Surg. 2012;41:113-8.

19. Benussi S, Galanti A, Zerbi V, Privitera YA, Iafelice I, Alfieri O Electrophysiologic efficacy of irrigated bipolar radiofrequency in the clinical setting. J Thorac Cardiovasc Surg. 2010;139:1131-6.

20. Tsui SS, Grace AA, Ludman PF, Schofield PM, Page AJ, Rowland E, et al. Maze 3 for atrial fibrillation: two cuts too few? Pacing Clin Electrophysiol. 1994;17: 2163-6.

21. Daccarett M, Badger TJ, Akoum N, Burgon NS, Mahnkopf C, Vergara G, et al Association of left atrial fibrosis detected by delayed-enhancement magnetic resonance imaging and the risk of stroke in patients with atrial fibrillation. $J$ Am Coll Cardiol. 2011;57:831-8.

22. Gasparovic H, Cikes M, Kopjar T, Hlupic L, Velagic V, Milicic D, et al. Atrial apoptosis and fibrosis adversely affect atrial conduit, reservoir and contractile functions. Interact Cardiovasc Thorac Surg. 2014;19:223-30.

23. Kim YH, Lee SC, Her AY, Kim HJ, Choi JO, Shin DH, et al. Preoperative left atrial volume index is a predictor of successful sinus rhythm restoration and maintenance after the maze operation. J Thorac Cardiovasc Surg. 2007;134: 448-53.

24. Damiano RJ Jr, Schwartz FH, Bailey MS, Maniar HS, Munfakh NA, Moon MR, et al. The Cox maze IV procedure: predictors of late recurrence. J Thorac Cardiovasc Surg. 2011;141:113-21.

25. Belus A, Piroddi N, Ferrantini C, Tesi C, Cazorla O, Toniolo L, et al. Effects of chronic atrial fibrillation on active and passive force generation in human atria myofibrils. Circ Res. 2010;107:144-52.

26. Rolf S, Kircher S, Arya A, Eitel C, Sommer P, Richter S, et al. Tailored atrial substrate modification based on low-voltage areas in catheter ablation of atrial fibrillation. Circ Arrhythm Electrophysiol. 2014;7:825-33.

Key Words: lesion set, posterior LA, transport function 